\title{
On the mechanism of deformation localization and homogeneous flow in bulk metallic glasses
}

\author{
J. C. $\mathrm{Li}^{1}$, X. W. Chen ${ }^{1} \&$ F. L. Huang ${ }^{2}$ \\ ${ }^{1}$ Institute of Systems Engineering, \\ China Academy of Engineering Physics, China \\ ${ }^{2}$ State Key Laboratory of Explosion Science and Technology, \\ Beijing Institute of Technology, China
}

\begin{abstract}
A modified coupled thermo-mechanical constitutive model for bulk metallic glasses (BMGs) is deduced in the present manuscript. Besides, a failure criterion of critical free volume concentration is introduced. The constitutive model as well as the failure criterion is implemented into the LS-DYNA commercial software through the user material subroutine (UMAT). Finite element method (FEM) simulations for different initial material temperatures and strain rates are conducted, and the evolutions of material parameter as well as the corresponding macroscopic mechanical behavior of material are analyzed. Related analysis shows that at room temperature, the deformation and failure characteristics in the shear band zones of BMGs are significantly different from that in the matrix, and consequently high deformation localization occurs. Comparatively, within the supercooled liquid region, the mechanical behaviors in the shear band zones and that in the matrix are similar to each other, and thus BMGs usually behave as homogeneous flow.

Keywords: bulk metallic glass, deformation, constitutive model, FEM simulation.
\end{abstract}

\section{Introduction}

Bulk metallic glasses (BMGs) have many excellent mechanical and physical performances. It usually shows good plasticity even superplasticity when under the condition of high temperature (within the supercooled liquid region) combined with low strain rate $[1,2]$. However, in the case of low temperature (below the glass transition temperature $T_{g}$ ) and high stress or high strain rate, highly localized 
shear bands are easily to be produced during the deformation process. Fracture occurs immediately along the propagation of shear bands and results in a catastrophic failure phenomenon. Moreover, the material usually shows an asymmetry between tension and compression [3,4]. All these particular properties promote that BMGs may have many engineering applications, e.g., the good plasticity is beneficial for the precise microforming process [5], and the localized shear bands may be employed in the material optimization of kinetic energy penetrator (KEP) [6-8].

Related analyses demonstrated that the microstructure of material dominates the deformation and failure of BMGs $[9,10]$. At present, a coupled thermomechanical shear model $[4,11]$, deriving from the free volume model $[9,10]$ and the adiabatic deformation theory [12], could predict the shear banding behavior of BMGs at room temperature relatively well. Comparatively, for the homogeneous flow of BMGs at high temperature ( $T \geq T_{g}$ ), the related analysis usually follows the models used for the crystalline alloys [1,2]. Regarding the yielding and failure of BMGs, some macroscopic failure criteria have been suggested [13, 14]. However, the corresponding micro-mechanism is still under the exploration process [15-17]. Recently, the simulation based on finite element method (FEM) is gradually employed for investigating the mechanical behavior of BMGs. Related work includes the theoretical constitutive modeling and the construction technique of the geometric model, etc. [18, 19].

The present manuscript further generalizes the coupled thermo-mechanical shear model into the multi-axial stress status, and takes all the contributions of free volume, temperature and hydrostatic stress into account. Besides, based on the coalescence mechanism of free volume in BMGs, a failure criterion of critical free volume concentration is introduced. Furthermore, by estimating related material parameters, the generalized multi-axial-stress-status model is extended to describe the mechanical behavior of BMGs at different deformation conditions. Then the constitutive model and failure criterion are implemented into LS-DYNA commercial software through the user material subroutine (UMAT), and FEM simulations for different initial material temperatures and strain rates are conducted. The evolutions of parameters as well as their effects on the mechanical behavior of material are analyzed, and the corresponding mechanism of deformation localization and homogeneous flow in BMGs is discussed.

\section{Constitutive model}

\subsection{Constitutive equations and failure criterion}

Based on the free volume model [9, 10], Dai and Bai [4] and Jiang and Dai [11] considered the effect of temperature additionally and suggested a coupled thermomechanical model for BMGs. However, the model is still limited within the onedimensional simple shear mode. Gao [18] generalized the one-dimensional free volume model into the multi-axial stress status but ignored the effect of temperature. Actually, during the deformation process of BMGs, the hydrostatic stress (or the normal stress acting on the shear plane), which derived from the 
shear-induced dilatation of material, also contributes to the deformation and failure $[3,13,14]$. Thus, by further considering the effect of hydrostatic stress, a modified thermo-mechanical model which is generalized to multi-axial stress status is obtained:

$$
\begin{gathered}
\dot{\varepsilon}_{i j}=\dot{\varepsilon}_{i j}^{e}+\dot{\varepsilon}_{i j}^{p} \\
\dot{\varepsilon}_{i j}{ }^{e}=\frac{1+v}{E}\left(\dot{\sigma}_{i j}-\frac{v}{1+v} \dot{\sigma}_{k k} \delta_{i j}\right) \\
\dot{\varepsilon}_{i j}^{p}=f \exp \left(-\frac{\Delta G^{m}}{K_{B} T}\right) \exp \left(-\frac{1}{\xi}\right) \sinh \left(\frac{\sigma_{e}^{\prime} \Omega}{2 K_{B} T}\right) \frac{S_{i j}}{\sigma_{e}} \\
\sigma_{e}=\sqrt{3 J_{2}} \\
\dot{\xi}=D \nabla^{2} \xi+\frac{1}{\alpha} f \exp \left(-\frac{\Delta G^{m}}{K_{B} T}\right) \exp \left(-\frac{1}{\xi}\right)\left\{\frac{2 K_{B} T}{\xi v^{*} S}\left[\cosh \left(\frac{\sigma_{e}^{\prime} \Omega}{2 K_{B} T}\right)-1\right]-\frac{1}{n_{D}}\right\} \\
\dot{T}=\kappa \nabla^{2} T+\frac{\beta_{T Q}}{\rho C_{v}} \sigma_{e}^{\prime} \dot{\varepsilon}_{e}^{p}
\end{gathered}
$$

In Eqs (1)-(3), a dot over a quantity $(\dot{)})$ denotes differentiation with time.

In Eq. (1) $E$ and $v$ are the Young's modulus and Poisson's ratio, respectively; $f$ is the frequency of atomic vibration and usually adopts the Debye frequency; $\Delta G^{m}$ is the activation energy; $K_{B}$ is the Boltzmann constant and $T$ the absolute temperature; $\xi=\bar{v}_{f} /\left(\alpha v^{*}\right)$ is the free volume concentration in BMGs (here $\bar{v}_{f}$, $v^{*}$ and $\alpha$ are, respectively, the average free volume per atom, critical volume (hard-sphere volume of an atom) and a geometrical factor) [10]; $\Omega$ is the average atomic volume; $\sigma_{e}$ is the von-Mises effective stress, in which $J_{2}=\sqrt{S_{i j} S_{i j} / 2}$ is the $J_{2}$ invariant of stress, $S_{i j}=\sigma_{i j}-\delta_{i j} \sigma_{m}$ is the deviatoric stress tensor and $\sigma_{m}=\sigma_{k k} / 3$ the mean stress; $\sigma_{e}^{\prime}$ is an effective stress which considers the contribution of hydrostatic stress, in which $\Lambda$ is the hydrostatic stress sensitivity factor.

Eq. (2) is the free volume evolution equation, in which $D$ is the diffusion coefficient of free volume; $s=E /[3(1-v)]$ is the Eshelby modulus and $n_{D}$ the number of diffusive jumps necessary to annihilate a free volume.

Eq. (3) is the temperature evolution equation, where $\kappa=K /\left(\rho C_{v}\right)$ is the thermal diffusivity (here $K, \rho$ and $C_{v}$ are the thermal conductivity, mass density and specific heat at constant volume, respectively); $\varepsilon_{e}^{p}=\sqrt{2 \varepsilon_{i j}^{p} \varepsilon_{i j}^{p} / 3}$ is the 
effective plastic strain. $\beta_{T Q}$ is the Taylor-Quinney coefficient which is usually taken as 0.9 or considered to be related to the strain rate [11]. Moreover, the analysis of Zhou et al. [20] demonstrated that when the material yields, the stress located within the shear band begins to collapse and unloads its neighboring material. Then the unloading wave propagates towards the neighboring zone of the shear band. When failure occurs in the shear band, the deformation energy in the neighboring zone where the unloading wave has arrived will release on the fracture surface and induces extreme temperature rise. Thus, in the simulation we could further modify the coefficient $\beta_{T Q}$ when the material is going to fail.

Furthermore, the related macroscopic failure criteria are difficult to describe the failure mechanism completely. Researchers analyzed the failure process based on the change of microstructure of BMGs $[15,16]$, and demonstrated that the free volume will be created during the deformation process and then coalesce together. Micro-voids will be formed due to the coalescence of free volumes and further leads to the damage and failure eventually. Thus, the failure criterion in the present model will be based on the evolution of microstructure. It is assumed that failure occurs when the net free volumes exceed a critical value, i.e. corresponding failure criterion is that the free volume concentration achieves the critical value $\xi_{c}$ :

$$
\xi \geq \xi_{c}
$$

\subsection{Finite element implementation of constitutive model}

Through the UMAT subroutine, the above constitutive model and the failure criterion are implemented into the LS-DYNA commercial software. As mentioned in [18], if the thermal conduction and the diffusion of free volume are both considered, it will be difficult to match up the two different length scales in the FEM simulation. As the influences of the diffusions of free volume and the thermal conduction are small $[10,11]$, to avoid the trouble of matching up the different length scales, the two diffusion issues are ignored in the FEM implementation. After such a predigesting treatment, the corresponding FEM simulation is independent on the length scale.

\subsection{Estimation of material parameters}

For convenience of comparative analysis with the corresponding test data, the typical $\mathrm{Zr}_{41.2} \mathrm{Ti}_{13.8} \mathrm{Cu}_{12.5} \mathrm{Ni}_{10.0} \mathrm{Be}_{22.5}$ (Vitreloy 1) BMG is chosen as the model material. Integrated with the related literature [10, 11, 15, 18-22], the material parameters are listed in Table 1 . The authors had conducted a detailed analysis on the parameters, and the related discussions can be referred in [22].

Especially, in the manufacture of BMGs an extremely rapid cooling process is necessary, and it usually induces some free volume in the inner structure of material. Thus, there exist some narrow weak zones which are usually called shear-band zone, where the initial free volume concentration $\xi_{0}$ is slightly larger than that in the matrix [21]. For comparative analysis, the initial free volume 
concentration in the matrix is adopted as $\xi_{0}=0.05$, and two kinds of shear-band zones, in which $\xi_{0}=0.051$ and 0.052 , respectively, are defined. Besides, to discuss the deformation and failure characteristics at the room temperature and within the supercooled liquid region, two typical initial temperature, $T_{0}=295 \mathrm{~K}$ and $643 \mathrm{~K}\left(>T_{g}\right)$, are selected for the corresponding analysis.

Table 1: $\quad$ Parameters of $\mathrm{Zr}_{41.25} \mathrm{Ti}_{13.75} \mathrm{Cu}_{12.5} \mathrm{Ni}_{10} \mathrm{Be}_{22.5}$ (Vitreloy 1) BMG.

\begin{tabular}{|c|c|c|c|}
\hline Parameter & Notation & Unit & Value \\
\hline \multirow{2}{*}{ Young's modulus } & \multirow{2}{*}{$E$} & \multirow{2}{*}{$\mathrm{GPa}$} & $96\left(<T_{g}\right)$ \\
\hline & & & $E\left(T_{0}, \dot{\varepsilon}\right)\left(\geq T_{g}\right)$ \\
\hline Poisson ratio & $v$ & - & 0.36 \\
\hline Glass transition temperature & $T_{g}$ & $\mathrm{~K}$ & 625 \\
\hline Melting temperature & $T_{m}$ & $\mathrm{~K}$ & 993 \\
\hline Density & $\rho$ & $\mathrm{kg} / \mathrm{m}^{3}$ & 6125 \\
\hline Specific heat at constant & $C_{v}$ & $\mathrm{~J} /(\mathrm{kg} \cdot \mathrm{K})$ & 400 \\
\hline Frequency of atomic vibration & $f$ & $\mathrm{~s}^{-1}$ & $1 \times 10^{13}$ \\
\hline Average atomic volume & $\Omega$ & $\mathrm{A}^{3}$ & 25 \\
\hline Critical volume & $v^{*}$ & $\mathrm{~A}^{3}$ & 20 \\
\hline Initial temperature & $T_{0}$ & $\mathrm{~K}$ & 295,643 \\
\hline Initial free volume concentration & $\xi_{0}$ & - & $0.05-0.052$ \\
\hline Critical free volume concentration & $\xi_{c}$ & - & 0.065 \\
\hline \multirow{2}{*}{ Geometrical factor } & \multirow{2}{*}{$\alpha$} & \multirow{2}{*}{-} & $0.05(295 \mathrm{~K})$ \\
\hline & & & $0.8(643 \mathrm{~K})$ \\
\hline Activation energy & $\Delta G^{m}$ & $\mathrm{eV}$ & $\Delta G^{m}(\dot{\varepsilon})$ \\
\hline \multirow{2}{*}{ Number of diffusive jumps } & \multirow{2}{*}{$n_{D}$} & \multirow{2}{*}{-} & $3(295 \mathrm{~K})$ \\
\hline & & & $6(643 \mathrm{~K})$ \\
\hline Hydrostatic stress sensitivity factor & $\Lambda$ & - & $\Lambda_{C}=0.05$ \\
\hline
\end{tabular}

\section{Deformation localization and homogeneous flow}

In this section the evolutions of various parameters under the different deformation conditions will be analyzed, and correspondingly the mechanism of deformation localization and homogeneous flow in BMGs is discussed.

For the computational convenience, a one-element geometrical model is adopted. As the characteristic size of the test sample is usually in millimeter scale, a hexahedral element with side length of $1 \mathrm{~mm}$ is chosen. Besides, for convenience to compare with corresponding test data, the uniaxial compressive deformation is considered, and two typical strain rates, $\dot{\varepsilon}=10^{-1} \mathrm{~s}^{-1}$ and $\dot{\varepsilon}=10^{4} \mathrm{~s}^{-1}$, are selected. The evolutions of various parameters under different conditions are shown in Figs 1-2, 
respectively, in which the abscissa represents the engineering strain. Besides, the test data are symbolized as "test" and indicate the test results in [2].

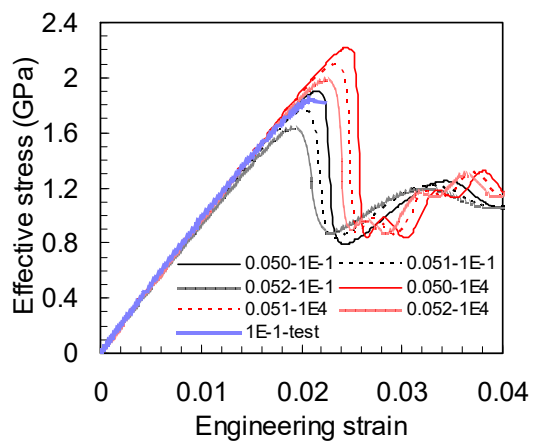

(a) Effective stress

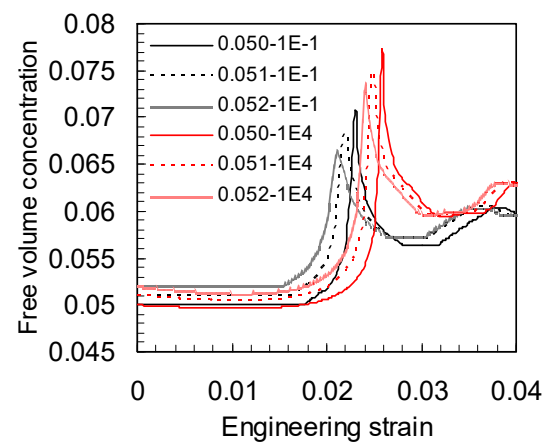

(b) Free volume concentration

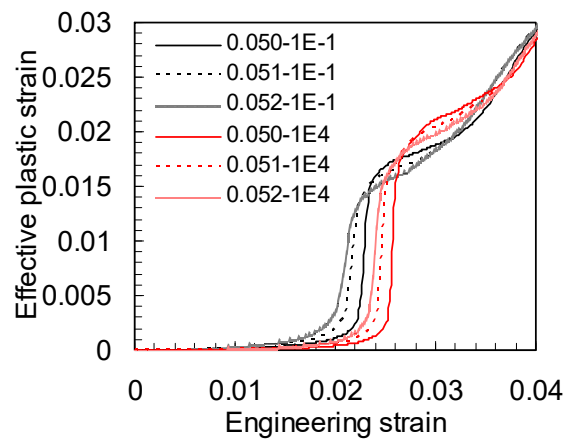

(c) Effective plastic strain

Figure 1: Evolutions of parameters for different values of $\xi_{0}$ under uniaxial compression at $T_{0}=295 \mathrm{~K}$ combined with different strain rates.

It can be seen from Figure 1 that the simulation results agree well with the corresponding test data. When under the condition of room temperature, the material begins to yield after the initial elastic response, and the corresponding stress drops precipitously, i.e. BMGs exhibit a significant strain softening after yielding. Then the stress shows a vibration after the collapse and further exhibits a slight strain hardening characteristic. Besides, it is found that with increasing the strain rate, the yielding stress increases, and the collapse of stress after softening becomes more significant.

In Figure 1(b)-(c) it is seen that the free volume concentration $\xi$ and the effective plastic strain $\varepsilon_{e}^{p}$ increase rapidly in the latter stage and show a precipitous increase characteristic, and the precipitous increase feature becomes 
more distinct with increasing the strain rate. Besides, it is found from the simulation results that the evolution of temperature $T$ is similar to that of effective plastic strain.

Especially, with the initial free volume concentration increases, the yielding stress $\sigma_{Y}$ and yielding strain $\varepsilon_{Y}$ decreases gradually. The weakest zone (with the largest $\xi_{0}$ ) yields first (see Figure 1(a)), and the rise of $\xi$ and $\varepsilon_{e}^{p}$ is earliest correspondingly (see Figure 1(b)-(c)). It implies that the onset of deformation localization and temperature rise will start in this zone, i.e. shear bands will nucleate in the weakest shear-band zone in BMGs. Besides, the time that $\xi$ rises up to the critical value $\xi_{c}$ is also earlier in the shear-band zone (see Figure 1(b)), indicating that failure will also start from this weak zone. After the shear-band zones yield or fail, the deformation or failure will locate in the corresponding shear bands. This may be the main reason of inhomogeneous deformation and failure in BMGs at the room temperature.

Regarding the case of $T_{0}=643 \mathrm{~K}$, as shown in Figure 2 , the simulation results also agree with the test data well. It is seen that under the low strain rate of $\dot{\varepsilon}=10^{-1} \mathrm{~s}^{-1}$, the yielding stress decreases a little compared with that at $T_{0}=295 \mathrm{~K}$, and the softening characteristic of stress becomes a little weaker. For the higher strain rates, the yielding stress increases rapidly and corresponding properties are more close to that at $T_{0}=295 \mathrm{~K}$ (see Figure 2(a)).

In Figure 2(b), at $\dot{\varepsilon}=10^{-1} \mathrm{~s}^{-1}, \xi$ decreases to a certain value in the early stage and then almost no longer changes during the latter stage, implying that the inner structure of material becomes more ordered. Comparatively, under the higher strain rate, the peak free volume concentration $\xi_{\text {peak }}$ in the latter stage exceeds the value of $\xi_{0}$, and it is more close to the case at room temperature.

Figure 2(c) shows that the effective plastic strain $\varepsilon_{e}^{p}$ increases slowly and smoothly during the deformation at $\dot{\varepsilon}=10^{-1} \mathrm{~s}^{-1}$, and the precipitous increase characteristic becomes more significant when the strain rate increases.

Thus, it can be seen that the deformation of BMGs depends much more strongly on the strain rate compared with the initial temperature. When under the high strain rate, the deformation at temperature of $T_{0} \geq T_{g}$ is similar to that at room temperature.

Moreover, it is found from Figure 2 that within the supercooled liquid region, the evolution of various parameters is almost the same for different $\xi_{0}$ at the strain rate of $\dot{\varepsilon}=10^{-1} \mathrm{~s}^{-1}$. In contrast, when $\dot{\varepsilon}=10^{4} \mathrm{~s}^{-1}$, the evolutions of various parameters are similar to that at the room temperature (see Figure 1). It explains the mechanism of homogeneous flow in BMGs under the condition of high temperature combined with low strain rate. Furthermore, it can be inferred that the inhomogeneous characteristic will become more significant gradually at the higher strain rate. 


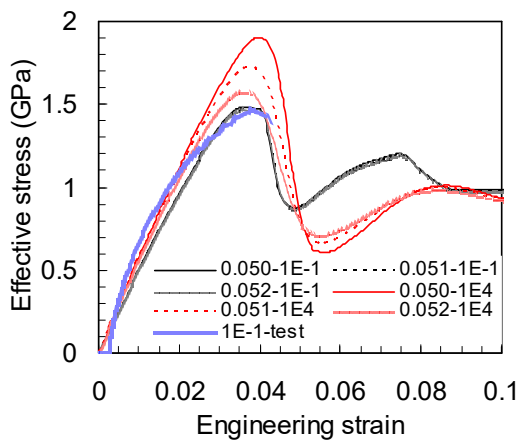

(a) Effective stress

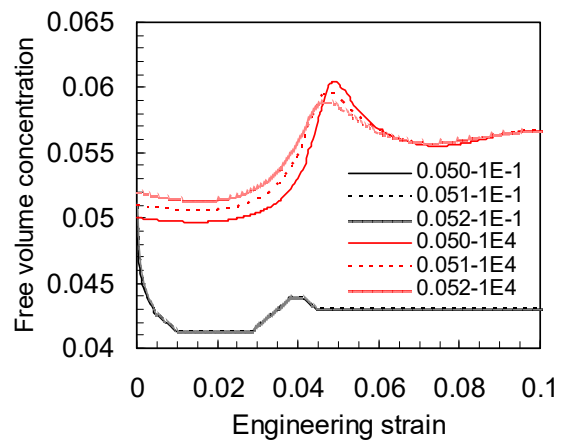

(b) Free volume concentration

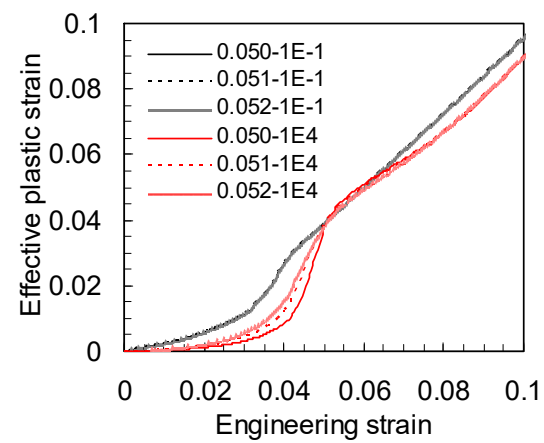

(c) Effective plastic strain

Figure 2: Evolutions of parameters for different values of $\xi_{0}$ under uniaxial compression at $T_{0}=643 \mathrm{~K}$ combined with different strain rates.

Consequently, it can be inferred that if $T_{0}<T_{g}$, the mechanical behavior in the shear-band zone and that in the matrix will be distinctly different and deformation localization occurs easily in BMGs. In contrast, under the condition of high temperature $\left(T_{0} \geq T_{g}\right)$ combined with low strain rate, the mechanical behavior in different zones are close to each other, and the deformation of BMGs usually behaves as homogeneous flow.

\section{Conclusions}

Based on the coupled thermo-mechanical model, a multiaxial-stress-status constitutive model which considers the effects of the free volume, temperature and hydrostatic stress on the deformation and failure of BMGs is further suggested in 
the present manuscript. Besides, a failure criterion of critical free volume concentration is introduced. The constitutive model and the failure criterion are implemented into the LS-DYNA commercial software through UMAT subroutine. Based on the FEM simulations, the corresponding mechanism of deformation localization and homogeneous flow in BMGs is studied.

Related analysis shows that both the strain rate and the initial temperature affect the mechanical behavior of BMGs, especially the influence of strain rate is significant. When under the condition of $T_{0}<T_{g}$, the material displays a precipitous softening of stress after yielding. The damage or crack nucleates in the shear-band zones and the nucleation time in various zones is different. Therefore, the deformation and failure process is very speedy and highly localized. In contrast, within the supercooled liquid region (i.e., $T_{0} \geq T_{g}$ ), the softening characteristic of material is weakened and the deviation of mechanical behavior between the shear-band zones and matrix reduces, the material prefers to flow homogeneously. However, the deformation will translate into inhomogeneous status if distinctly increasing the strain rate.

\section{Acknowledgements}

The project is supported by the Science and Technology Development Foundation (2015B0201025) as well as the key subject "Computational Solid Mechanics" of China Academy of Engineering Physics, the National Outstanding Young Scientists Foundation of China (11225213) and the National Natural Science Foundation of China (11521062).

\section{References}

[1] Inoue, A., Kawamura, Y., Shibata, T., et al. Viscous flow deformation in supercooled liquid state of bulk amorphous $\mathrm{Zr}_{55} \mathrm{Al}_{10} \mathrm{Ni}_{5} \mathrm{Cu}_{30}$ alloy. Materials Transactions JIM, 39 (6), pp. 1337-1341, 1996.

[2] Lu, J., Ravichandran, G., Johnson, W.L. Deformation behavior of the $\mathrm{Zr}_{41.2} \mathrm{Ti}_{13.8} \mathrm{Cu}_{12.5} \mathrm{Ni}_{10} \mathrm{Be}_{22.5}$ bulk metallic glass over a wide range of strainrates and temperatures. Acta Materialia, 51, pp. 3429-3443, 2003.

[3] Zhang, Z.F., Eckert, J., Schultz, L. Difference in compressive and tensile fracture mechanisms of $\mathrm{Zr}_{59} \mathrm{Cu}_{20} \mathrm{Al}_{10} \mathrm{Ni}_{8} \mathrm{Ti}_{3}$ bulk metallic glass. Acta Materialia, 51, pp. 1167-1179, 2003.

[4] Dai L.H., Bai Y.L. Basic mechanical behaviors and mechanics of shear banding in BMGs. International Journal of Impact Engineering, 35, pp. 704-716, 2008.

[5] Nishiyama, N., Inoue, A. Glass transition behavior and viscous flow working of $\mathrm{Pd}_{40} \mathrm{Cu}_{30} \mathrm{Ni}_{10} \mathrm{P}_{20}$ amorphous alloy. Materials Transactions JIM, 40 (1), pp. 64-70, 1999.

[6] Conner, R.D., Dandliker, R.B., Scruggs, V., et al. Dynamic deformation behavior of tungsten-fiber / metallic-glass matrix composites. International Journal of Impact Engineering, 24, pp. 435-444, 2000. 
[7] Rong, G., Huang, D.W., Yang, M.C. Penetrating behaviors of Zr-based metallic glass composite rods reinforced by tungsten fibers. Theoretical and Applied Fracture Mechanics, 58, pp. 21-27, 2012.

[8] Chen, X.W., Wei, L.M., Li, J.C. Experimental research on long rod penetration of tungsten fiber/Zr-based metallic glass matrix composite into Q235 steel target. International Journal of Impact Engineering, 79, pp. 102-116, 2015.

[9] Spaepen, F. A microscopic mechanism for steady state inhomogeneous flow in metallic glasses. Acta Metallurgica, 25 (4), pp. 407-415, 1977.

[10] Huang, R., Suo, Z., Prevost, J.H. Inhomogeneous deformation in metallic glasses. Journal of the Mechanics and Physics of Solids, 50, pp. 1011-1027, 2002.

[11] Jiang, M.Q., Dai, L.H. On the origin of shear banding instability in metallic glasses. Journal of the Mechanics and Physics of Solids, 57, pp. 1267-1292, 2009.

[12] Liu, C.T., Heatherly, L., Easton, D.S., et al. Test environments and mechanical properties of Zr-base bulk amorphous alloys. Metallurgical and Materials Transactions A, 29A, pp. 1811-1820, 1998.

[13] Zhang, Z.F., Eckert, J. Unified tensile fracture criterion. Physical Review Letters, PRL 94, pp. 094301-1-4, 2005.

[14] Chen, Y., Jiang, M.Q., Wei, Y.J., et al. Failure criterion for metallic glasses. Philosophical Magazine, 91(36), pp. 4536-4554, 2011.

[15] Wright, W.J., Hufnagel, T.C., Nix, W.D. Free volume coalescence and void formation in shear bands in metallic glass. Journal of Applied Physics, 93 (3), pp.1432-1437, 2003.

[16] Xue, Y.F., Cai, H.N., Wang, L., et al. Effect of loading rate on failure in Zrbased bulk metallic glass. Materials Science and Engineering A, 473, pp. 105-110, 2008.

[17] Jiang, M.Q., Ling, Z., Meng, J.X., et al. Energy dissipation in fracture of bulk metallic glasses via inherent competition between local softening and quasi-cleavage. Philosophical Magazine, 21(3), pp. 407-426, 2008.

[18] Gao, Y.F. An implicit finite element method for simulating inhomogeneous deformation and shear bands of amorphous alloys based on the free-volume model. Modelling and Simulation in Materials Science and Engineering, 14, pp. 1329-1345, 2006.

[19] Chen, Y., Jiang, M.Q., Dai, L.H. How does the initial free volume distribution affect shear band formation in metallic glass?. Science China: Physics, Mechanics \& Astronomy, 54 (8), pp. 1488-1494, 2011.

[20] Zhou, F., Wright, T.W., Ramesh, K.T. A numerical methodology for investigating the formation of adiabatic shear bands. Journal of the Mechanics and Physics of Solids, 54, pp. 904-926, 2006.

[21] Steif, P.S., Spaepen, F., Hutchinson, J.W. Strain localization in amorphous metals. Acta Metallurgica, 30, pp. 447-455, 1982.

[22] Li, J.C., Wei, Q., Chen, X.W., et al. On the mechanism of deformation and fracture in bulk metallic glasses. Materials Science and Engineering A, 610, pp. 91-105, 2014. 